\title{
REPÚBLICA DOMINICANA, ¿DEMOCRACIA SIN ESTADO DE DERECHO?
}

\author{
DOMINICAN REPUBLIC, \\ DEMOCRACY WITHOUT THE RULE OF LAW? \\ REPÚBLICA DOMINICANA, \\ DEMOCRACIA SEM ESTADO DE DIREITO?
}

Elissa Loraine Lister Brugal

Doctora en Literatura Hispanoamericana

Universidad Nacional de Colombia

Coordinadora grupo de trabajo CLACSO

Afrodescendencia, racismo y resistencias en el Caribe (2016-2019)

elister@unal.edu.co

Colômbia

\section{Resumen}

Ensayo de Elissa Loraine Lister Brugal.

Palabras clave: Republica Dominicana, democracia, estado de derecho

\section{Abstract}

Essay by Elissa Loraine Lister Brugal.

Keywords: Dominican Republic, democracy, rule of law

\section{Resumo}

Ensaio de Elissa Loraine Lister Brugal.

Palavras-chave: República Dominicana, democracia, estado de direito 


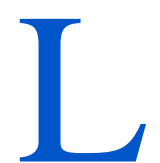

a pregunta en el título de este trabajo surge a partir de la historia política de República Dominicana y la atención que ocupa en la actualidad lo inédito de tres procesos electorales, no libres de polémica, en un periodo de menos de ocho meses. Éstos son la celebración de primarias de los partidos mayoritarios el 6 de octubre de 2019; la jornada de elecciones municipales del 16 de febrero de 2020, suspendidas y anuladas luego de iniciadas las votaciones, $\mathrm{y}$ las elecciones presidenciales y del Congreso previstas para el 17 de mayo del año en curso. En el centro del debate se han mantenido la actuación y decisiones de quienes dirigen la Junta Central Electoral (JCE), su cuestionable dependencia y adscripción al poder ejecutivo y el empeño para imponer el voto electrónico en los comicios referidos en un contexto de firme intención continuista del Partido de la Liberación Dominicana (PLD), que ha gobernado ininterrumpidamente por 16 años.

La implementación fallida del voto electrónico en dos de las elecciones en cuanto a garantizar la expresión de la voluntad ciudadana, el derecho de elegir y ser elegido, el secreto del voto, la confiabilidad y legitimidad de los resultados, así como la posibilidad de rotación de autoridades y de representantes, han sido los detonantes de la fragmentación y reordenamiento de las fuerzas políticas tradicionales y del surgimiento de la movilización y protesta de amplios sectores de la sociedad, en especial de los jóvenes. El propósito de este texto es desarrollar algunas observaciones y análisis en torno a estos procesos sociopolíticos y su significación y función en lo que podría ser la construcción de un Estado de derecho, en su sentido más amplio, justo e inclusivo.

El antecedente inmediato de los procesos que nos ocupan lo constituye la promulgación de la primera Ley de partidos, agrupaciones y movimientos políticos en República Dominicana (Ley 33-18), el 15 de agosto de 2018. Durante casi dos décadas habían resultado infructuosas las iniciativas legislativas para dotar al país de esta regulación. Entre las novedades que introdujo la normativa figura la organización de primarias simultáneas por parte del ente electoral para los partidos que se acojan a esta modalidad de selección de candidatos presidenciales.

El 6 de octubre de 2019 se efectuaron las primeras primarias en el país, participando el Partido Revolucionario Moderno (PRM) en votación con padrón cerrado y el PLD, con padrón abierto. El resultado que favoreció al candidato vencedor del partido gobernante (PLD) fue puesto en entredicho con reiteradas denuncias de fraude digital por su contrincante Leonel Fernández, presidente del país en tres periodos (1996-2000, 20042008 y 2008-2012) y aspirante una vez más. Las impugnaciones interpuestas por el 
afectado en los órganos electorales no prosperaron. El impasse por la cuestionada derrota interna motivó la salida de Fernández del partido que había dirigido por 18 años y la postulación de su candidatura a la presidencia bajo nuevas siglas.

Las dudas en torno al voto electrónico se extendieron, generando que las diferentes agrupaciones políticas acordasen con la JCE que en las elecciones regionales de 2020 se aplicarían las dos modalidades de votación: el sufragio electrónico en los municipios que concentraran el mayor número de votantes (sumando el 62\%) y el manual, en los demás. Esta simultaneidad de formas de votación, por sí sola, resulta cuestionable y hace evidentes la debilidad institucional, las ambivalencias políticas, los acomodos normativos y las complacencias múltiples.

La convocatoria para las elecciones municipales del 16 de febrero de 2020 estuvo atravesada por continuas denuncias de compra de votos, propaganda ilícita, competencia desleal, uso de los recursos públicos en beneficio de las campañas oficialistas y acciones de intimidación a candidatos y votantes opositores, prácticas que no cesan. El día de la jornada electoral los

El domingo 15 de marzo de 2020 tuvieron lugar las elecciones municipales extraordinarias, que se realizaron mediante el voto manual en el $100 \%$ del territorio nacional y sin aparentes contratiempos logísticos. La noche antes, el sábado 14 de marzo, el gobierno nacional hizo públicas una serie de medidas tomadas contra la pandemia del coronavirus. Si bien no se decretó en esa fecha el aislamiento obligatorio, la abstención en los ciudadanos y ciudadanas se vieron impedidos de ejercer el sufragio. Algunas mesas de voto manual no contaban con los materiales necesarios; en el caso del voto electrónico, la boleta omitía candidatos o mostraba exclusivamente a los del PLD. Muchos de los equipos no entraron en funcionamiento, permaneciendo cerradas las mesas de votación. La JCE suspendió el proceso trascurridas 4 horas de iniciado aduciendo "fallas técnicas", al día siguiente decidió su reprogramación para un mes después, el 15 de marzo. Se perdían de este modo los cerca de 160 millones de dólares del erario público utilizados en la realización de los comicios.

Al momento de escribirse esto, la sociedad dominicana continúa sin recibir una versión oficial de lo ocurrido el 16 de febrero por parte de las autoridades electorales. Éstas han continuado ejerciendo sus funciones plenamente, sin identificar responsabilidades y responsables, libres de un régimen de consecuencias políticas, económicas y penales, en tanto han proseguido al frente de la organización de los comicios de marzo y de mayo ${ }^{1}$.

Los acontecimientos anteriores se adicionan a una historia política dominicana

comicios fue de aproximadamente el 66\%. De acuerdo con los informes de Participación Ciudadana, el capítulo dominicano de Transparencia Internacional, se registraron numerosos delitos electorales asociados a compra de votos y proselitismo electoral en los centros de votación que involucraban a candidatos tanto del partido en el gobierno como de los de oposición. 
en la que la manipulación y el fraude en las elecciones parecen ser la norma y no la excepción. Están asociados también a las concepciones autocráticas del ejercicio del poder y del Estado que practican la mayor parte de los gobernantes dominicanos y sus agrupaciones políticas, en el que se naturaliza su permanencia indefinida y la apropiación de los recursos públicos. El término democracia se instaura, entonces, como un significante vacío empleado a conveniencia bajo ciertos formalismos.

Entre estos últimos el más representativo es la realización periódica de elecciones y el supuesto ejercicio del sufragio. Muchos de estos procesos se han efectuado sin las garantías necesarias, bajo condiciones de represión, intervención de intereses foráneos, con candidato y partido único u oposición desde el exilio, además del fraude. Así ocurrió durante la dictadura de 31 años (1930-1961) de Rafael Leonidas Trujillo, en la que sistemáticamente se efectuaban elecciones. Su ideólogo y heredero político, Joaquín Balaguer, continuó esta práctica durante el denominado Régimen de los 12 años (19661978) y prosiguió con algunos matices en sus gobiernos posteriores (1986-1990, 19901994, 1994-1996). Llegado el siglo XXI se incorpora a lo anterior la modalidad de las reformas constitucionales "a pedido" en torno a la reelección (2002, 2010 y 2015).
Esto ha permitido al PLD ejercer el dominio del país desde 1996 hasta el presente (a excepción del intervalo de 2000 a 2004) con la alternancia de tan solo dos nombres: Leonel Fernández y Danilo Medina.

Paralelamente a lo descrito se registra en la sociedad dominicana una tradición de resistencia y lucha en pos de valores democráticos que ha sido combatida desde los sectores hegemónicos tanto con la violencia como con el silencio y el olvido impuestos a través de las políticas de la memoria. De este modo, en los últimos 40 años los integrantes de los grupos de poder se han promovido como los garantes "naturales, legítimos y únicos" de la democracia y el orden ante coyunturas de posibles crisis y de movilización social que son generadas, casi siempre, por las acciones y políticas impulsadas por estas mismas élites en su beneficio $y$ en detrimento de la institucionalidad, las condiciones de vida $\mathrm{y}$ derechos de los ciudadanos. Integrantes de la cúpula política de turno, de la dirigencia empresarial, de la jerarquía de la Iglesia y los representantes del gobierno de los Estados Unidos establecen pactos y acuerdos cerrados de los cuales se excluye la participación y representación de los demás sectores y organizaciones de la sociedad. Por lo general, dichos pactos suelen vulnerar y superponerse a lo decidido por la ciudadanía en las urnas o mediante la manifestación popular. Sirven 
como ejemplo lo acordado durante la “transición” política en 1978 para finalizar el Régimen de los 12 años de Joaquín Balaguer, en el que el nuevo gobierno quedó limitado en su ejercicio por un Congreso de mayoría balaguerista, o el fraude electoral de 1994, que le impidió el acceso a la presidencia a José Francisco Peña Gómez.

En años más recientes encontramos lo sucedido a partir del movimiento que se produjo entre 2010 y 2012 en favor de la inversión del $4 \%$ del producto interno bruto para la educación, como lo establecía la constitución. En 2012, en plena campaña a la presidencia, el candidato Danilo Medina se comprometió con dar curso a este mandato. Ya en el poder los recursos del $4 \%$ para este sector se instrumentalizaron y desvirtuaron en una extensa red clientelista y de corrupción. La Iglesia católica se benefició de estas dinámicas al firmar en 2015 un convenio con el Ministerio de Educación por el cual las escuelas y colegios de su propiedad pasaban a ser costeados por el Estado, incluida la formación y actualización de sus docentes. Así, el derecho a la educación universal y de calidad continúa siendo una deuda y la "Revolución educativa" del gobierno, una

Cf. ORGANISATION FOR ECONOMIC COOPERATION AND DEVELOPMENT (OECD). PISA 2018 Results (Volume I): What Students Know and Can Do. Paris: OECD, 2019. Disponible en https://doi.org/10.1787/5f07c754-en. Consultado el 7 de marzo de 2020.

3 El nombre Odebretcht corresponde a una compañía constructora brasileña que recibió numerosos contratos falsa propaganda: en 2018 la República Dominicana obtuvo los peores resultados (“por debajo del nivel") en las denominadas pruebas PISA (Programme for Internatinal Student Assessment) con relación a los 79 países evaluados ${ }^{2}$.

La coyuntura actual evidencia el declive del PLD, así como su empeño para conservar el poder a toda costa y garantizar una impunidad sin plazo de vencimiento. Baste recordar que en el caso de corrupción trasnacional de Odebrecht $^{3}$, la República Dominicana ocupó el tercer lugar como mayor receptora de los pagos ilícitos, la oficina encargada de la distribución de los sobornos internacionales de la compañía operaba en el país, la justicia dominicana no adscribió el pacto de colaboración entre fiscalías y no hay condenados en firme en el proceso.

En el PLD hizo carrera lo postulado por Leonel Fernández durante sus mandatos en cuanto a que el partido era una "fábrica de presidentes" y que gobernarían hasta el 2044, año del bicentenario de la independencia. Amparados en esta máxima han practicado la corrupción sin límites y sin disimulos, los abusos de poder, la continua vulneración de los derechos fundamentales de la ciudadanía

para megaobras de infraestructura de carácter público en Brasil, América Latina y Africa. En el 2016 el Departamento de Justicia de los Estados Unidos hizo pública su investigación sobre las prácticas ilegales y corruptas de la compañía para obtener las contrataciones en asociación con gobernantes, candidatos y sectores empresariales de cada uno de los países donde actuaba. 
y el uso de la fuerza, la censura y la represión en diferentes formas y niveles. Consecuencia de lo anterior es la agudización de las desigualdades y de la pobreza, además de la violencia. Los datos del Banco Mundial indican que el promedio de crecimiento del producto interno bruto (PIB) en América Latina en la década 2000-2010 fue del 2.9\%. La República Dominicana sostuvo dicho promedio en $3.8 \%$. La gran diferencia estriba en que mientras la tasa de movilidad social de la región fue de un $41 \%$, en el país solo un $2 \%$ pudo ingresar a un grupo económico más alto; un $19 \%$ de la población dominicana descendió en la escala económica y el 79\% permaneció en la misma condición. Esta inequidad entre bonanza macroeconómica y mínima redistribución se mantiene y ha sido reiterada en informes de los años sucesivos, aun cuando el PIB continuara su crecimiento.

Este contexto, sumado a los sucesos electorales recientes, ha acentuado el descontento de la población, que se materializa en la voluntad y demanda pública y sostenida de cambio de gobierno, democratización e institucionalidad. Frente a estos reclamos también se ven abocados a dar respuesta los partidos mayoritarios de la oposición: el Partido Revolucionario Moderno (PRM), encabezado por Luis Abinader, que se vislumbra como virtual ganador en las encuestas, y Leonel Fernández, que se presenta bajo Fuerza del
Pueblo (FP) luego de renunciar al PLD. Convenientemente, desde estas agrupaciones se capitaliza e instrumentaliza la expectativa de cambio de la ciudadanía. La noción de democracia se ha equiparado a alternancia de partido político en la dirección del Estado, así que promueven a sus respectivos candidatos como la encarnación misma del anhelado cambio.

El interés de acceder al poder $y$ desplazar al PLD ha llevado al PRM y FP a acuerdos entre estos, así como a alianzas con múltiples partidos minoritarios y organizaciones políticas con los más disímiles intereses y orientaciones ideológicas. Las alianzas y pactos tienen como función principal sumar votos, no están mediados por compromisos y acuerdos programáticos que garanticen el Estado de derecho. De este modo, parece aplicarse el principio de que "el fin justifica los medios". En lo que concierne a los electores, parecen estar dispuestos a entregar su voto como especie de cheque en blanco.

Otra función que cumplen estas alianzas consiste en revivir agrupaciones y partidos prácticamente extintos, habilitándolos para acceder a curules y recibir fondos públicos para sus campañas y organizaciones. Esta estrategia, en un primer término, tiene como objetivo modificar el mapa político en cuanto a los 
gobiernos locales y terminar con la mayoría absoluta del PLD en el Congreso, buscando mayor gobernabilidad para un nuevo partido en el poder. Una panorámica sobre este conjunto de agrupaciones permite observar, en un segundo término, que bajo el falso discurso de la pluralidad y la apertura se estaría diseñando un congreso en que las fuerzas conservadoras y más retardatarias se afianzarían y ampliarían con una mayor representatividad.

Ocurre también que los sectores hegemónicos tradicionales se colocan otra vez en la primera línea, garantizando su ejercicio de poder de forma directa en instancias del Estado y ya no a través de negociaciones y acuerdos tácitos con el PLD como partido de gobierno. Una muestra de esto se observa en la elección de las candidatas vicepresidenciales de los partidos de oposición PRM y FP. De este modo las posibilidades de construcción de un Estado de derecho parecen alejarse. Quienes respaldan y protagonizan la baraja electoral detentan -parcial o colectivamente, directa o indirectamenteniveles de responsabilidad en el régimen de vulneración de derechos que impera en el país, que incluye las violencias ejercidas desde el nacionalismo, la proliferación de políticas y prácticas racistas y antihaitianas, ubicar a la República Dominicana como el quinto país con mayor apatridia, la casi "imposibilidad" de descriminalizar el aborto en al menos tres causales, la expropiación de tierras en centros urbanos y rurales, la violación continua a los derechos de los inmigrantes haitianos, la entrega de los recursos naturales a las industrias mineras y del turismo trasnacional, así como la prohibición de facto de programas sobre equidad de género en la educación básica y media, entre muchas otras acciones que niegan el reconocimiento y ejercicio de derechos a la población.

Otro aspecto que también pone en entredicho la materialización real de cambio lo constituye el transfuguismo político, práctica habitual que se acentúa en el periodo de elecciones. En la coyuntura actual, en la medida que las encuestas y la movilización social inclinan la balanza hacia el triunfo del PRM, funcionarios del PLD y políticos aliados de éste se declaran a favor y partidarios de Luis Abinader (PRM). Así mismo, peledeístas y de otros partidos emigran a los toldos de Leonel Fernández (FP). Estas dinámicas constatan el clientelismo y oportunismo que ejercen tanto quienes trasmutan de siglas, pretendiendo desligarse del desprestigio, de la estela de corrupción y de las responsabilidades políticas, sociales e, incluso, judiciales de su quehacer, como los dirigentes que los reciben en sus 
agrupaciones y le proveen una especie de "reciclaje" y "cuenta nueva".

Estas maniobras no escapan a la consciencia y mirada crítica de sectores de la población sobre los intereses políticos en juego y los poderes en disputa, en los que la noción de transformaciones estructurales y la construcción del Estado de derecho están ausentes. El texto de una pancarta exhibida en las manifestaciones de la Plaza de la Bandera el 20 de febrero probablemente lo exprese con un claro principio de realidad (o, si se quiere, pesimismo): "Los políticos son iguales, la gente no es boba, pero nos quieren quitar el derecho a elegir a quien nos roba".

En este escenario hace presencia el movimiento social como un nuevo actor político. La cancelación de las elecciones municipales del 16 de febrero podría considerarse el detonante de esta movilización que por espacio de 12 días, con protagonismo de los jóvenes, de manera continua y cada vez más numerosa, ocupó la plaza pública en la capital y múltiples ciudades, acaparó la atención de los medios de comunicación y sorprendió e interpeló a las autoridades gubernamentales.

Un primer momento tuvo lugar con la vigilia que realizaron integrantes y seguidores del partido opositor Alianza País ante las instalaciones de la JCE, en la Plaza de la Bandera, la noche de las elecciones “fallidas". El lunes 17 de febrero se dieron cita en el mismo lugar estudiantes procedentes de universidades privadas de élite de Santo Domingo. El lanzamiento de 3 bombas lacrimógenas a los manifestantes el martes 18, sumado a la ausencia de respuesta institucional ante lo sucedido en las elecciones, hizo que a partir del miércoles 19 se incorporaran masivamente a la protesta personas provenientes de los más diversos sectores y que la participación fuera en aumento con el paso de los días. La movilización se extendió a las plazas de ciudades y capitales de provincia. Se introdujo la modalidad del cacerolazo; calderos, ollas y sartenes resonaron en barrios, calles, centros comerciales, restaurantes y espacios de reunión. También se multiplicaron los escraches a políticos y candidatos del oficialismo.

Democracia, cambio y verdad eran reiterados en pancartas y consignas en reclamo de conocer lo ocurrido, demandar la renuncia de la JCE, proclamar la salida del poder del PLD y exigir garantías en el ejercicio del derecho al voto. El momento culminante de la movilización ocurrió en la ciudad de Santo Domingo el 27 de febrero de 2020, día de la conmemoración de la Independencia Nacional. En esta fecha, mientras el presidente Danilo Medina realizaba la rendición de cuentas de su gestión ante el Congreso, más de 100,000 personas acudían a la emblemática Plaza de 
la Bandera en repudio a la burla electoral y reclamo de garantías institucionales para los comicios pendientes.

La salida a la plaza pública de los jóvenes y demás sectores de la sociedad mostró un descontento e indignación que ya no era posible contener o convencer mediante la propaganda y las múltiples estrategias de cooptación del oficialismo. Expresó la decisión contundente de la población en torno a aspiraciones de cambio de gobierno y a una apertura más democrática.

Cuando se intenta analizar este proceso, que se considera novedoso en el contexto político dominicano, se incurre en dos prácticas que suelen ser cuestionadas desde ciertos estudios sobre los movimientos sociales: desconocer la memoria y tradición de la movilización social, asumiendo que se trata de algo inédito y surgido exclusivamente "al calor del momento", y considerar al colectivo que se moviliza como un todo homogéneo, en este caso bajo la categoría de "jóvenes"4.

Con relación a lo primero, es preciso indicar que la movilización reciente se articula a una tradición de acción y lucha en pro de derechos múltiples ejercida por

Cf. SÁNCHEZ RAMOS, Irene. "Sujetos sociales: historia, memoria y cotidianeidad". In: SÁNCHEZ RAMOS, Irene; SOSA ELÍZAGA, Raquel (coordinadoras.) América Latina: los desafíos del pensamiento crítico. México: Siglo XXI, 2004. p.219250; REGUILLO, Rossana. "Las culturas juveniles: un campo de estudio; breve agenda para la discusión". Revista Brasileira de Educação (23), 2003, p. 103-118; diversos colectivos, organizaciones $\mathrm{y}$ espacios de confluencia, principalmente en los ámbitos urbanos, que convocan en torno a problemáticas y realidades, y en los que la experiencia directa o indirecta propicia una toma de consciencia, conocimiento y perspectiva crítica sobre las mismas. Puede darse a través de grupos de música urbana, comités barriales, asociaciones estudiantiles, entes gremiales, religiosos, deportivos, de defensa del ecosistema y los recursos naturales, cooperativismo, emprendimiento, entre otros posibles de una larga lista. Estos colectivos integraron de forma importante el movimiento de febrero de la Plaza de la Bandera. En lo que concierne a los sectores más vulnerables, su participación en el espacio público les posibilitó vencer el cerco del que muchas veces son objeto sus protestas y reclamos cuando se les criminaliza, descalifica y reprime en los escenarios en que normalmente actúan.

Probablemente apeló a esta memoria de los movimientos, al devenir político dominicano, a la historicidad de las realidades y/o a su experiencia de vida un joven manifestante que de manera

JIMÉNEZ GUZMÁN, Lucero (coordinadora). Jóvenes en movimiento en el mundo globalizado. México: UNAM, 2016; FLÓREZ FLÓREZ, Juliana. "Los movimientos sociales y la crisis del desarrollismo: una aproximación teórica desde Latinoamérica". Buenos Aires: CLACSO, 2009. 
contundente declaró en su pancarta: “Tengo tatuajes, no amnesia".

El sesgo clasista, racista y etario con el que generalmente se concibe en el país el derecho al ejercicio político, en el que solo unos estarían llamados a ejercer el gobierno y la representación, mientras al grueso de la población se le culpa por el sistema fallido, tiende a invisibilizar o negar la trascendencia y continuidad del activismo social y político de más larga data y desde sectores no-hegemónicos. La reiterada afirmación en medios de comunicación dominicanos de que se trató de un "movimiento de jóvenes de clase media y alta" se constituye en expresión del sesgo referido y patentiza las prácticas de discriminación y exclusión múltiples, incluso, en el derecho a la protesta. La complejidad del proceso conlleva tener en cuenta los múltiples actores, sus roles y funciones y lo cambiante de las dinámicas, interrelaciones y rumbos en un espacio de tiempo muy breve.

En lo que remite a la práctica de homogenizar los sujetos de los movimientos sociales, hay que señalar que los jóvenes que se expresaron y expresan en la plaza pública son tan diversos $\mathrm{y}$ heterogéneos como lo es la sociedad dominicana. Análisis amplios y profundos requieren que lo etario se intersecte con la clase, el género, lo racial, la educación, la religión, la adscripción ideológica, el trabajo, para dar cuenta de la diversidad de la composición del movimiento. La confluencia de variables posibilita identificar tanto los puntos en común que viabilizaron lo de febrero, como las diferencias y divergencias en cuanto a propósitos, posiciones políticas, continuidad o no en la movilización, las formas de lucha y los derechos que se reivindican.

Los jóvenes manifestantes que procedían de los sectores más pudientes enfatizaron en sus convocatorias por redes y medios, sobre todo en los primeros días, que su protesta era "apolítica", pretendiendo tomar distancia de lo partidario. En la concentración del 27 de febrero integrantes de este sector leyeron la "Proclama por la democracia y el derecho al voto". Los contenidos y demandas estuvieron alineados con lo que simultáneamente solicitaban los partidos de oposición mayoritarios -el PRM y la FP-, en cuanto a que la Organización de Estados Americanos (OEA) y la Fundación Internacional de Sistemas Electorales (IFES) efectuaran la investigación sobre lo ocurrido en las elecciones y ejercieran de veedores y garantes de los comicios pendientes, entre otros puntos. Luego del evento la mayoría de este grupo se desmovilizó. 
Un posicionamiento y perspectiva política diferentes lo expresó el colectivo La Plaza Alzá. El 25 de febrero, dos días antes de la masiva concentración, difundieron un manifiesto en el que denunciaban el carácter injerencista de la OEA y proclamaban el derecho de autodeterminación para dirimir los asuntos políticos del país. Se desmarcaron así de la postura del primer grupo. Incluían, además, críticas al sistema político en su conjunto (no sólo al PLD), postulaban la equidad, rechazaban el racismo y demandaban el derecho a la salud y a la educación. Dicho manifiesto evidenció el interés de trascender la coyuntura y mantener la movilización en torno al reclamo de un Estado de derecho.

Somos Pueblo y Creo en ti hacen parte de los colectivos que se aglutinaron en torno a algunos intereses compartidos con $\mathrm{La}$ Plaza Alzá y que continuaron movilizados con posterioridad al 27 de febrero. Las concentraciones y protestas masivas dieron paso a la realización periódica de asambleas, reuniones y manifestaciones puntuales en la Plaza de la Bandera con el objetivo de desarrollar acciones de pedagogía política. Asimismo, se sucedieron escraches y denuncias frente a centros en los que se pretendían realizar pactos entre los sectores tradicionales de las élites a espaldas de la ciudadanía. En contraposición, integrantes del movimiento social organizaron el $6 \mathrm{de}$ marzo un diálogo político con participación de los partidos, instituciones ciudadanas y movimientos sociales. Los jóvenes reivindicaban así formas alternativas e inclusivas para construir consenso y en las que tuviesen una participación activa en las instancias de discusión y toma de decisiones del quehacer político.

Como puntos en común entre los dos grupos descritos están la reafirmación generacional, el reclamo democrático (aunque entendido de forma diferente) y el compromiso de velar por el proceso electoral ejerciendo de delegados de partidos, facilitadores de la JCE, veedores $u$ observadores en las mesas de votación del 15 de marzo.

Ante la importancia y extensión que han tenido los movimientos sociales en América Latina en el último año, resulta necesario plantear algunos matices $y$ diferencias significativas con relación a lo ocurrido en República Dominicana en febrero de 2020. En las movilizaciones y protestas en otros países del continente se suelen recuperar nombres, sucesos o fechas emblemáticos asociados a la tradición de lucha de los colectivos. De este modo se apela a memorias subalternas que son continuamente actualizadas. Esto hace parte de un amplio proceso de construcción simbólica y resimbolización que le es propio a los movimientos. En el país, en 
cambio, esto no ocurrió. Predominó el empleo de las insignias nacionales, la continua alusión a los "Padres de la Patria" y la exaltación del término patria y lo patriótico. En muchas pancartas se reprodujeron frases y pensamientos que se atribuyen al patricio Juan Pablo Duarte, considerado fundador de la nación. Queda abierta la pregunta de si esto pudo deberse a lo arraigado de la ideología nacionalista en la sociedad dominicana, que emplea estos mismos recursos, o al hecho que la movilización se inició en los días previos a la conmemoración de la Independencia y tuvo su apogeo en esta fecha.

Así mismo, mientras las reivindicaciones de género se incorporan como un eje transversal de los movimientos sociales actuales, la misma estuvo ausente en lo llevado a cabo en la media isla. No está de más recordar que República Dominicana se encuentra entre los 5 países de la región con mayor índice de feminicidios y ocupa el primer lugar de América Latina y el Caribe en matrimonio infantil (36-38\%), lo que genera una alta tasa de embarazo adolescente.

Otra diferencia significativa se observa en que, por lo general, la movilización estudiantil y juvenil en Chile, Colombia y México, por sólo mencionar algunas, tiende a solidarizarse con otras causas y luchas vigentes, que también hacen propias. De este modo, a las demandas específicas del colectivo, se integran las de otros sectores como maestros, campesinos, grupos étnicos, comunidad LGBTI. En República Dominicana esta solidaridad no se ejerció o no se hizo visible en febrero.

Lo descrito anteriormente permite afirmar que los sesgos en la concepción de democracia, en la que el término se disocia de la construcción de un Estado de derecho, están vigentes también en el imaginario y en las prácticas de los sectores que mayoritariamente integraron el movimiento de la Plaza de la Bandera.

Esta noción de democracia formal resulta instrumentalizada desde el gobierno y otras instancias de poder. A la creencia de que hay democracia porque hay elecciones, adicionan el supuesto de que esta se verifica porque también hay protesta social y el derecho a la libre expresión. Pero lo cierto es que ni la reivindicación de derechos ni la expresión son tan libres ni para todos o todas. En el espacio público en que se abogó en febrero por la democracia, se hicieron presentes también el racismo, las desigualdades consolidadas en la sociedad y el Estado dominicanos y las prácticas de coacción vigentes.

Caso significativo es el del movimiento Reconoci.do, integrado por jóvenes dominicanos a quienes el Estado privó de su nacionalidad en 2013 por ser descendientes de inmigrantes haitianos (son 
cerca de 210,000 afectados). Como ha ocurrido en movilizaciones anteriores, sus integrantes acudieron a las manifestaciones desafiando la intimidación y múltiples amenazas a su integridad física. $\mathrm{Su}$ ejercicio ciudadano en la protesta se instaura, entonces, como experiencia de nuevas vulneraciones y suma de violencias.

Por el contrario, los sectores nacionalistas reafirmaron su presencia en las manifestaciones con pancartas racistas $\mathrm{y}$ antihaitianas. Entre ellos, la Antigua Orden Dominicana -un grupo "pseudoparamilitar" o "paramilitar a lo dominicano"- hizo despliegue abierto en diferentes momentos de la movilización, incluida la multitudinaria concentración del 27 de febrero. El grupo, que actúa con el beneplácito de las autoridades, se autodefine como una "organización cívica" que bajo el lema de "patria o muerte" difunde impunemente un discurso de odio racista y antihaitiano, hace incitación a la violencia y profiere amenazas y sentencias explícitas en redes, medios de comunicación y actos públicos. Bajo el mismo lema incluye también en sus ataques a los periodistas que denuncian la corrupción y son críticos con el gobierno. Justifica su comparecencia en la movilización como el ejercicio de un pretendido derecho ciudadano bajo el que encubre la función de infiltrar y desvirtuar la protesta.
Hasta el momento no se la responsabiliza de desapariciones o asesinatos. En el contexto dominicano esas acciones la ejecutan abiertamente los integrantes de las fuerzas del orden, principalmente la policía. Los informes anuales de la Comisión Nacional de Derechos Humanos, Amnistía Internacional y la Organización de Naciones Unidas (ONU) de las últimas tres décadas registran que cada año se producen entre 150 y 400 ejecuciones extrajudiciales, sumarias o arbitrarias cometidas por la Policía Nacional. Dichos informes señalan también la recurrencia a la tortura en los recintos policiales y la impunidad de que gozan estas violaciones.

La coyuntura política analizada no estuvo exenta de expresiones de los abusos $\mathrm{y}$ arbitrariedades de la fuerza policial. La exigencia de la ciudadanía y los partidos políticos para obtener explicaciones sobre lo que originó los "desperfectos" en la aplicación del voto electrónico y la consiguiente anulación de los comicios el 16 de febrero, ubicar responsables y aplicar régimen de consecuencias, unido a la repercusión en los medios internacionales de lo acontecido, se constituyeron en mecanismos de presión interna y externa para los organismos del Estado, no habituados a rendir cuentas y justificar sus acciones. La respuesta inmediata de la Policía Nacional y el Ministerio Público fue 
culpabilizar y usar como chivos expiatorios a dos personas que habían alertado sobre "manipulación irregular" de equipos en la JCE la noche previa a las elecciones. Se trató de un coronel de la Policía, asignado como escolta del candidato opositor Luis Abinader (PRM), y un técnico de una compañía de telecomunicaciones que brinda servicios a la JCE. Los afectados sufrieron captura y detención ilegal, apresamiento sin debido proceso y, en el caso del técnico, torturas evidentes. Las flagrantes violaciones fueron denunciadas por instituciones de Derechos Humanos. Fue necesaria una orden del Poder Ejecutivo, el 21 de febrero, para suspender la rocambolesca "investigación". En ese momento estaba en juego la integridad de los dos hombres, así como desmontar la trama oficialista que pretendía acusar al PRM del sabotaje de las elecciones.

La situación anterior muestra el accionar de las fuerzas del orden dentro del contexto amplio de violación de los derechos fundamentales en el país y da cuenta, desde otro ámbito, de la continuidad de prácticas instauradas durante la dictadura trujillista. No obstante, el militarismo y la brutalidad policial continúan dejándose de

Cf. CENTRO PARA LA OBSERVACIÓN MIGRATORIA Y EL DESARROLLO SOCIAL EN EL CARIBE (OBMICA). Estado de las migraciones que atañen a la República Dominicana 2017. Santo Domingo: Búho, 2018; DIARIO LIBRE. "Aumentan a 2.1 millones los dominicanos en Estados Unidos", 21 de noviembre de 2018. Disponible en lado en los debates en torno a la noción de democracia y del Estado de derecho, tanto por parte de los partidos políticos como en la movilización social.

Los análisis sobre los procesos sociopolíticos en el país deben, necesariamente, incluir la dimensión diaspórica de la sociedad dominicana, generada por la masiva emigración y asentamiento de nacionales en diferentes países y continentes. La comunidad dominicana en los Estados Unidos representa la más numerosa y de mayor tradición. Según ciertas estadísticas, se estima que cuenta con cerca de 2,100,000 integrantes, de los cuales el 56\% nació en la isla y el $44 \%$, en territorio de la unión. España es el segundo destino de la diáspora, con aprox. 165,000 personas, siendo el mayor porcentaje de mujeres. Puerto Rico, Italia, Suiza, Holanda, Canadá, Islas Vírgenes, Panamá y Chile constituyen otros centros importantes de recepción de los migrantes dominicanos ${ }^{5}$.

Como consecuencia, "la dominicanidad" se vive y expresa desde múltiples localizaciones que trascienden $\mathrm{y}$ distan de las fronteras insulares. La identidad dominicana adquiere una extensión

https://www.diariolibre.com/actualidad/aumentan-a-2-1millones-los-dominicanos-en-estados-unidosFA11444919. Consultado el 14 de marzo de 2020. GALLUP. Gallup World Poll. 2018. Disponible en https://www.gallup.com/analytics/247355/gallup-worldhappiness-report.aspx\#contactus. Consultado el 14 de marzo de 2020. 
trasnacional que la hace aún más diversa y heterogénea, lo que en la práctica conlleva a dejar de lado los esencialismos tan del gusto de los puristas y nacionalistas. En lo que respecta al ejercicio ciudadano de la diáspora, hay que precisar que este adquiere una concepción y práctica pluriestatal pues puede ejercerse tanto en el país de acogida como en el de origen. La aprobación de la doble nacionalidad en las reformas a la constitución en 1994 fue el primer paso para la habilitación política de los migrantes dominicanos en el país; continuó con el voto en el exterior a partir de las elecciones del 2004, y se consolidó en 2012 con la elección de 7 curules en el Congreso Nacional por circunscripción en el exterior.

Lo que en un primer término puede parecer un reconocimiento de derechos a los dominicanos residentes en el extranjero representa, simultáneamente, la inmersión de esta población en la lógica y distorsión de una democracia que no se concibe más allá del formalismo. Las cifras de migrantes, unido a que el $49 \%$ de la población desea marcharse, revelan las inequidades del sistema y la ausencia de un Estado de derecho. En la República Dominicana el estado de bienestar no es concebido como un compromiso y política estatal, sino como una responsabilidad que se traslada a los individuos. Son estos quienes lo deben generar y garantizar para ellos y su núcleo familiar. Para un buen número de dominicanos y dominicanas, las condiciones existentes niegan resultados mínimos en estos esfuerzos, por lo que la opción es la salida. De este modo, la migración ha operado en las últimas cuatro décadas como especie de válvula de escape a la presión social que se pudiera generar por las desigualdades, la precariedad del mercado laboral, la carencia en los servicios y las necesidades básicas insatisfechas en el país. Hoy, las remesas que envían desde el exterior quienes emigran representan más del 8\% del PIB de la República Dominicana, constituyen la tercera fuente de ingresos de divisas a la economía nacional y suman un monto que supera con creces la inversión en programas de asistencia social del gobierno. Datos aportados por el Banco Interamericano de Desarrollo en 2004 indicaban que alrededor del $38 \%$ de los adultos del país recibían remesas.

El modelo económico se perpetúa, entonces, con ventajas. Las plazas vacantes en las áreas productivas que requieren menos cualificación (construcción, agricultura, servicios) son ocupadas por los inmigrantes haitianos, quienes desempeñan las labores en condiciones de mayor vulnerabilidad y precariedad.

Las responsabilidades económicas de la diáspora dominicana, la participación política en procesos electorales, así como 
los vínculos y conexiones con sus familias y localidades, le proporcionan un conocimiento actualizado y de primera mano del acontecer diario del país. Así mismo, el hecho de que se trate de economías interdependientes -en su dimensión local, estatal y global- hace más susceptible a esta comunidad para movilizarse en sus respectivos contextos ante acontecimientos de la realidad dominicana. Establecen así un diálogo trasnacional en la protesta en el que ejercen la vocería para la difusión internacional de ciertas problemáticas y, simultáneamente, aportan dinámicas de concienciación política, experiencias de organización y movilización que los colectivos y movimientos en el país pueden capitalizar para su desarrollo. En febrero, ciudades como Nueva York, Miami, Washington, San Juan de Puerto Rico, Toronto, París y Santiago de Chile, entre otras, fueron escenarios de las protestas masivas de dominicanas y dominicanos que reivindicaron democracia, elecciones transparentes, justicia, cambio, pero que recurrieron también a su experiencia y memoria como migrantes para realizar críticas más amplias al sistema.

A modo de cierre, sostengo que los movimientos sociales que lograron un amplio poder de convocatoria en el pasado reciente en República Dominicana al interpelar a diferentes sectores de la sociedad, generaron amplias -y quizás exageradas y dudosas- expectativas de cambio: La Plaza de la Bandera (2020) surgió en torno a la reivindicación del derecho al voto y elecciones transparentes y su antecedente inmediato, Marcha Verde (2017), condenó la corrupción y la impunidad tras hacerse público el caso Odebrecht. Si bien abogaron por principios de democracia, justicia e institucionalidad, silenciaron u obviaron lo concerniente al racismo estructural, las desigualdades multidimensionales, el nacionalismo, el patriarcado, el antihaitianismo, la no laicidad en el Estado, entre otros. En la práctica estas reivindicaciones resultaron sesgadas, parciales $\mathrm{y}$, probablemente, contradictorias por asumir la defensa de un derecho excluyendo, negando e, incluso, vulnerando otros.

La construcción de un Estado de derecho implica, necesariamente, que el reconocimiento y respeto a los derechos fundamentales se conciba de una forma integral, coherente y universal y se coloque en el centro de las propuestas y programas de los partidos; de los planes, políticas y leyes que se implementan desde el Estado, $\mathrm{y}$ de las causas y reivindicaciones que movilizan a la sociedad.

Plantear los derechos de la misma forma como se han asumido 
tradicionalmente las nociones de cambio y democracia en la República Dominicana no hace más que garantizar el establishment, continuar en el juego de los formalismos que se propagan desde el poder y postergar y enmascarar el debate en torno a las transformaciones estructurales que se requieren en el país.

Por otro lado, si se tiene en cuenta la interrelación entre el sistema político parcialmente descrito (sin garantías de derechos), el modelo económico de servicios (sustentado en la emigración-inmigración y mano de obra poco cualificada y de mínimo salario) y el diseño social (en función del racismo y las desigualdades), el panorama se presenta complejo y con infinidad de dudas e incertidumbres acerca de la factibilidad de transformaciones estructurales en el corto, mediano o largo plazo. Así, debatiéndonos entre lo que puede actuar como sentencia o desafío, entre el presente y el futuro, retomamos la pregunta "República Dominicana, ¿democracia sin Estado de derecho?". 